\title{
Light Scattering by Cholesteric Skyrmions
}

\author{
G. De Matteis $\dagger^{*} \quad$ D. Delle Side $\dagger^{\dagger} \quad$ L. Martina $\dagger_{\dagger}^{\ddagger} \quad$ V. Turco $\dagger^{\S}$ \\ * IISS "V. Lilla", MIUR, Francavilla Fontana (BR) Italy \\ †Dipartimento di Matematica e Fisica, Università del Salento \\ $\ddagger$ INFN, Sezione di Lecce \\ Via per Arnesano, C.P. 193 I-73100 Lecce, Italy
}

June 19, 2018

\begin{abstract}
We study the light scattering by localized quasi planar excitations of a Cholesteric Liquid Crystal known as spherulites. Due to the anisotropic optical properties of the medium and the peculiar shape of the excitations, we quantitatively evaluate the cross section of the axis-rotation of polarized light. Because of the complexity of the system under consideration, first we give a simplified, but analytical, description of the spherulite and we compare the Born approximation results in this setting with those obtained by resorting to a numerical exact solution. The effects of changing values of the driving external static electric (or magnetic) field is considered. Possible applications of the phenomenon are envisaged.
\end{abstract}

\section{Introduction}

In the last few years great efforts have been done in developing new materials for opto-electronics and photonics applications. A relevant role in this work has been played by the liquid crystals (LC) physics 1.2 for a quite long time. In fact, nowadays LC are widely used in all types of display applications and their unique nonlinear electro-optical properties make them suitable material for non-display applications, like optical filters and switches, beam-steering devices, spatial light modulators, optical wave-guiding, lasers 3 and optical nonlinear components 4 . On the other hand, a wide interest was deserved by a variety of new 2-dimensional structures like cholesteric fingers 5, and 3-dimensional ones, like nematicons [6, cholesteric bubbles or spherulites [7 9], the latter appear in quasi-2D layers of Chiral Liquid Crystals (CLCs) with homeotropic anchoring on the confining surfaces. Those textures have been studied from a theoretical point of view 10,11 and we would like to consider them for their potential opto-technological applications. Thus the aim of the present paper is to evaluate the possibility to exploit spherulites, isolated or in lattice arrangements 12, 13, as electric/magnetically driven switches for light beams, propagating in the liquid crystal.

Spherulites in CLCs share some properties with the 2D skyrmions in magnetic systems [14,15]. In fact, these isolated axisymmetric states are stabilized by specific interactions imposed by the underlying molecular handedness, however they are more sensible to the external fields and may possess slow modulations in a preferred direction. Thus, a continuum model can be derived in the framework of the Frank-Oseen theory [16 17, from which one can write the respective equilibrium equations. By applying external fields and imposing anchoring boundary conditions [18 19], the free helicoidal equilibrium can be deformed into new structures such as skyrmions 20,21, which are stabilized by topological conservation laws. The theory also describes the cholesteric fingers 22 23], or helicoids, with defect disclination type, which can be described, at least in some approximate setting, in terms of integrable nonlinear equations 12,24 , stabilized both by topological and non-topological conservation laws. Carboni et al. [13] detected a phase transition between the two textures, strongly depending on the thickness of the confining

\footnotetext{
*e-mail: giovanni.dematteis@istruzione.it

†e-mail:nico.delleside@unisalento.it

$\ddagger$ e-mail:martina@le.infn.it

§e-mail: vito.turco@live.com
} 
cell. They showed that the texture changes are driven by temperature through a parameter $\zeta$ proportional to the thickness and to a proper chirality parameter. Samples of different thickness displayed the textural changes at different temperatures but for the same value of $\zeta$. However here we limit ourselves to the sferulites/skyrmion case.

The paper is organized as follows. In Sec. 2 we introduce the continuum elastic model of the CLC, we obtain the corresponding equilibrium equations and analyse the skyrmion (spherulite) solutions, either by analytical or numerical methods. In Sec. 3 we introduce the problem of light diffusion by a spherulite. In Sec. 4 we provide perturbative solutions for the light scattering equations derived in Sec. 3 . In particular in 4.1 we compute the cross section of the conversion process of incoming polarized light in the incidence plane into the outgoing polarized light in the perpendicular direction. Analogously, in 4.2 we consider the complementary problem of the change of polarization axis from the direction orthogonal to the liquid crystal to the in plane direction. Finally, in the Conclusions we summarise our results and address some possible experimental realizations.

\section{Skyrmions in chiral liquid crystals}

A LC is described by a uni-modular director field $\mathbf{n}(\mathbf{r})$ belonging to $\mathbb{R P}^{2}$ 16, 17], which in polar representation is

$$
\mathbf{n}(\mathbf{r})=(\sin \theta(\mathbf{r}) \cos \psi(\mathbf{r}), \sin \theta(\mathbf{r}) \sin \psi(\mathbf{r}), \cos \theta(\mathbf{r})), \quad-\mathbf{n} \equiv \mathbf{n} .
$$

In the bulk a CLC director field $\mathbf{n}(\mathbf{r})$ is governed by the Frank-Oseen free energy density

$$
\begin{array}{r}
\omega_{F O}=\frac{K_{1}}{2}(\nabla \cdot \mathbf{n})^{2}+\frac{K_{2}}{2}\left(\mathbf{n} \cdot \nabla \times \mathbf{n}-q_{0}\right)^{2}+\frac{K_{3}}{2}(\mathbf{n} \times \nabla \times \mathbf{n})^{2} \\
+\frac{\left(K_{2}+K_{4}\right)}{2} \nabla \cdot[(\mathbf{n} \cdot \nabla) \mathbf{n}-\nabla \cdot \mathbf{n}-(\nabla \cdot \mathbf{n}) \mathbf{n}]-\frac{\varepsilon}{2}(\mathbf{n} \cdot \mathbf{E})^{2},
\end{array}
$$

where $q_{0}$ is the chirality parameter of the cholesteric phase, the positive reals $K_{1}, K_{2}, K_{3}, K_{4}$ are the Frank elastic constants, which we set to be $K=K_{1}=K_{2}=K_{3}, \quad K_{4}=0$ for sake of simplicity. The last term in 2.2 represents the interaction energy density associated with a spatially uniform external static electric field $\mathbf{E}$, or equivalently a magnetic field $\mathbf{H}$, along the $\mathbf{k}$ direction. Of course, in the presence of the external electric (magnetic) field, the general rotational symmetry is broken and reduced to rotations around the direction of $\mathbf{E}(\mathbf{H})$. In the absence of anchoring conditions, the field $\mathbf{n}(\mathbf{r})$ would form a cholesteric helix with axis orthogonal to $\mathbf{E}(\mathbf{H})$. However, supposing the CLC confined within the region $\mathcal{B}=\left\{(x, y, z) \in \mathbb{R}^{3},|z| \leq \frac{L}{2}\right\}$, the translational symmetry in the direction of $\mathbf{k}$ is broken and the interaction of the CLC with the planar bounding surfaces can be encoded by the Rapini and Papoular 25 additional surface energy contribution

$$
\omega_{s}=\frac{1}{2} K_{s}\left(1+\alpha(\mathbf{n} \cdot \boldsymbol{\nu})^{2}\right)
$$

where $K_{s}, \alpha>0$ and $\boldsymbol{\nu}$ being the unit outward normal to the boundary surface. Strong homeotropic anchoring is obtained for $K_{s} \rightarrow \infty$, which corresponds to the Dirichlet boundary conditions $\mathbf{n}\left(x, y, z \pm \frac{L}{2}\right)=$ $\mathbf{k} \equiv-\mathbf{k}$. So helices are deformed and confined within $\mathcal{B}$ and possibly extended structures called helicoids (or helicons and, sometimes, fingers) or spherulites (also skyrmions) may form, depending on the existence of a preferred direction of perturbations of $\mathbf{n}$.

In order to find equilibrium configurations of the CLC we have to minimise the Frank free energy under the appropriate boundary conditions. We also limit ourselves to axisymmetric isolated solutions. Thus, assuming $\theta=\theta(\rho, z)$ and $\psi=\psi(\phi)$, where $\rho, z$ and $\phi$ are the usual cylindrical coordinates around the axis $\mathbf{k}$, the solution of minimal energy is given by

$$
\psi(\phi)=\phi+\frac{\pi}{2}, \quad \phi \in[0,2 \pi]
$$

and all the admissible equilibrium configurations are solutions of the dimensionless Boundary Value 
Problem (BVP)

$$
\begin{aligned}
\frac{\partial^{2} \theta}{\partial z^{2}}+\frac{\partial^{2} \theta}{\partial \rho^{2}}+\frac{1}{\rho} \frac{\partial \theta}{\partial \rho}- & \frac{1}{\rho^{2}} \sin \theta \cos \theta \mp \frac{4 \pi}{\rho} \sin ^{2} \theta-\pi^{4}\left(\frac{E}{E_{0}}\right)^{2} \sin \theta \cos \theta=0, \\
& \left\{\begin{array}{c}
\theta(0, z)=\pi, \quad \theta(\infty, z)=0, \\
\partial_{z} \theta\left(\rho, \pm \frac{\nu}{2}\right)=\mp 2 \pi k_{s} \sin \theta\left(\rho, \pm \frac{\nu}{2}\right) \cos \theta\left(\rho, \pm \frac{\nu}{2}\right),
\end{array}\right.
\end{aligned}
$$

where the lengths are rescaled with respect to the so-called pitch length $p=\frac{2 \pi}{\left|q_{0}\right|}$. Here, $E_{0}=\frac{\pi\left|q_{0}\right|}{2} \sqrt{\frac{K}{\varepsilon}}$ is the critical unwinding field for the cholesteric-nematic transition in non-confined CLCs [26], $\nu=L / p$ is the normalized thickness of the layer and $k_{s}=K_{s} /\left(K q_{0}\right)$ the strength of the interaction liquid/ boundary surfaces. The $\mp$ sign in equation (2.5) depends on the sign of $q_{0}$ : in the following we take $q_{0}<0$, with no loss of generality. Moreover, it is convenient to simplify the notation setting $\rho_{1}^{2}=\pi^{4}\left(\frac{E}{E_{0}}\right)^{2}$. System (2.5 2.6) is a 3D perturbed Sine-Gordon type equation: chirality and BCs do not allow to integrate it in analytical form. The main deformation comes from the fifth term in 2.5), associated to the chirality of the system. Thus, the solutions of the BVP (2.5 2.6) can be obtained, at least to our knowledge, only by numerical methods.

However, to get information about the shape of a spherulite, one can evaluate the asymptotic behaviours of the solutions near $\rho \rightsquigarrow 0$ and $\rho \rightsquigarrow \infty$. Moreover, let us consider first the pure cylindrical reduction of 2.5), i.e. $\theta_{z}=0$, which holds when $\nu$ is sufficiently large and modulations in the $z$ variable are discarded.

Near $\rho \rightsquigarrow 0$ both the chiral and the electric interaction can be neglected with respect to the other terms, thus setting both $q_{0} \rightarrow 0$ and $E \rightarrow 0$, equation (2.5) reduces to the the conformally invariant $\mathrm{O}(3)$-sigma model in polar representation [27. Accordingly, the solutions near $\rho \rightsquigarrow 0$ behave like the Belavin-Polyakov ones [28, namely

$$
\theta=\arccos \left(\frac{\tilde{\rho}^{2}-4}{\tilde{\rho}^{2}+4}\right), \quad \tilde{\rho}=\frac{\rho}{\rho_{0}}
$$

where $\rho_{0}$ is an arbitrary scale factor due to the conformal invariance. The fourth and the fifth term in 2.5 break the conformal symmetry. Thus, substituting solution 2.7 in equation 2.5 we obtain the extimation

$$
\rho_{0}=\frac{4}{\pi^{3}}\left(\frac{E_{0}}{E}\right)^{2}=4 \pi \rho_{1}^{2},
$$

which can be interpreted as the typical scale of a spherulite. Then, around $\rho=0$, at the lowest order the solution of 2.52 .6 is approximated by

$$
\theta(\rho)=\pi-\frac{\rho}{\rho_{0}}+O\left(\left(\frac{\rho}{\rho_{0}}\right)^{3}\right) .
$$

with $\rho_{0}$ fixed by (2.8). Furthermore, in order to have information also about the modulation in the $z$ direction, we assume as rough approximation of the solution by deforming $(2.9)$ as

$$
\theta(\rho, z)=\left\{\begin{array}{cc}
\pi-\frac{\rho}{\rho_{0} Z(z)} & \rho / Z(z)<\pi \rho_{0} \\
0 & \rho / Z(z)>\pi \rho_{0}
\end{array}\right.
$$

with $\rho_{0}$ given by (2.8), and replace 2.10 into the Frank-Oseen energy. Its minimisation leads to equation

$$
Z^{\prime \prime}(z)-\frac{1}{\pi^{2} \rho_{1}^{2}} Z(z)+\frac{1}{\pi^{2} \rho_{1}^{2}}=0,
$$

which, upon imposing the boundary conditions $(2.6)$, yields the solution

$$
Z(z)=1-\frac{2 \pi k_{s} \cosh \left(\frac{z}{\rho_{1}}\right)}{2 \pi k_{s} \cosh \left(\frac{1}{\rho_{1}} \frac{\nu}{2}\right)+\frac{1}{\rho_{1}} \sinh \left(\frac{1}{\rho_{1}} \frac{\nu}{2}\right)} .
$$




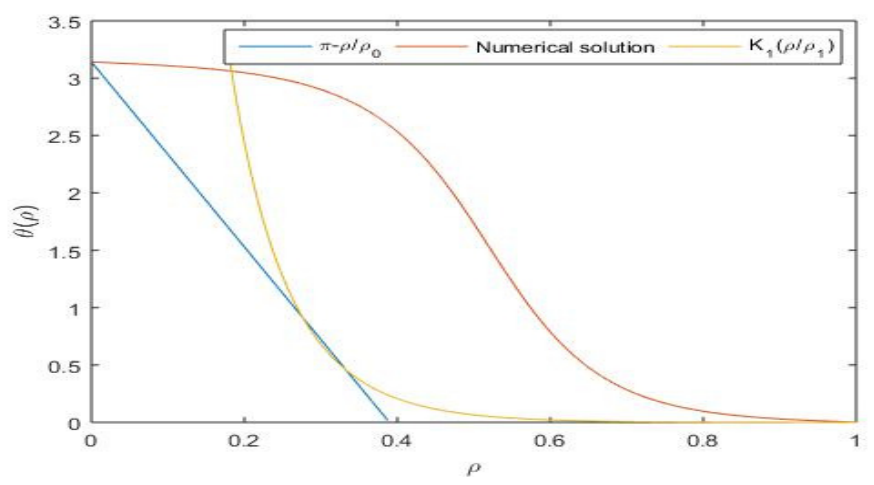

Figure 1: Comparison between the numerical solution of (2.5) and the analytical linear approximations for $\frac{E}{E_{0}}=1.02$.

We note that the sizes of the vortices decrease as $|z|$ and $k_{s}$ increase, as it can be seen in figure 3 . We will assume hereafter that this is the $z$-modulation of the skyrmion in the entire volume.

In the asymptotic limit $\rho \rightarrow \infty$ the dominant term comes from the external electric field, which affects the shape of Skyrmion by the reduced equation

$$
\frac{\partial^{2} \theta}{\partial \rho^{2}}+\frac{1}{\rho} \frac{\partial \theta}{\partial \rho}-\frac{\rho_{1}^{2}}{2} \sin 2 \theta=0,
$$

which is known as cylindrical Sine-Gordon equation 29]. The most relevant fact about this equation is its connection with the celebrated Painlevé III equation [11,30,31 (see also 32 Cap. 32): then it can be analytically solved. However, in correspondence to the boundary conditions at $\infty$ stated in $(2.6)$, this equation has always singular solutions at $\rho \rightarrow 0$. Thus the validity of such an approximation is limited to a neighbourhood of $\infty$, where its asymptotics is

$$
\theta \rightsquigarrow c_{2} \sqrt{\frac{\rho_{1}}{\rho}} \exp \left[-\frac{\rho}{\rho_{1}}\right] .
$$

This result is sufficiently similar to the one obtained in linear approximation, which leads to first order modified Bessel functions of second kind which have almost analogous asymptotics.

The above results give us useful indications about the shape of the spherulite/skyrmion, but many important details are missed. In fact, to have a good account of them and to estimate the goodness of the approximations made above, we need to perform numerical calculations on the BVP described by 2.5 . 2.6). To this aim, we use the standard central finite difference discretisation and the Newton-Raphson method 33, 34], inizialized by the shooting method for the planar reduction of the system (i.e. $\theta_{z}=0$ ).

It turns out that for sufficiently large electric fields, i.e. $\frac{E}{E_{0}}>1$ the linear approximations matches with the numerical solution quite closely, as represented in fig. 2. On the other hand, the approximations become very rough for relatively weak fields, i.e. $\frac{E}{E_{0}} \approx 1$, as shown in fig. 2, As far as the numerical cases considered in the present work, this behaviour denotes the underestimation of the chiral term in the linear approximation, in particular at the intermediate scales $\rho_{1} \leq \rho \leq \rho_{0}$.

The numerical solutions of the BVP 2.5$)$, for different values of the couple $\left(\frac{E}{E_{0}}, k_{s}\right)$ are depicted in figures 4 and 5 . In each figure the profiles $\theta(\rho)$ for different values of $z \in[-\nu / 2, \nu / 2]$ are represented. In figure 4 we have $\frac{E}{E_{0}}=1.02$ and the strength of the anchoring $k_{s}=0.1,6$. In figure 5 we have $\frac{E}{E_{0}}=1.5$ with the same values of $k_{s}$. We note that, when the strength of the anchoring is small, the profiles are almost the same for every value of coordinate $z$. This means that, when the interfaces at the boundaries of the cell have a really small homeotropic effect on the director's configuration, a quasi-perfect cylindrical simmetry holds for axisymmetric solutions. In this case, the planar vortices described by $\theta(\rho)$ for every value of $z$, have the same, maximum, size. However, if we impose a quite stronger homeotropic effect at the boundaries, the vortices tend to have a reduced size, which becomes smaller as $|z|$ reaches the value $\frac{\nu}{2}$. In both figures 4 and 5 the value of the adimensional thickness of the cell is $\nu=1.8$. 


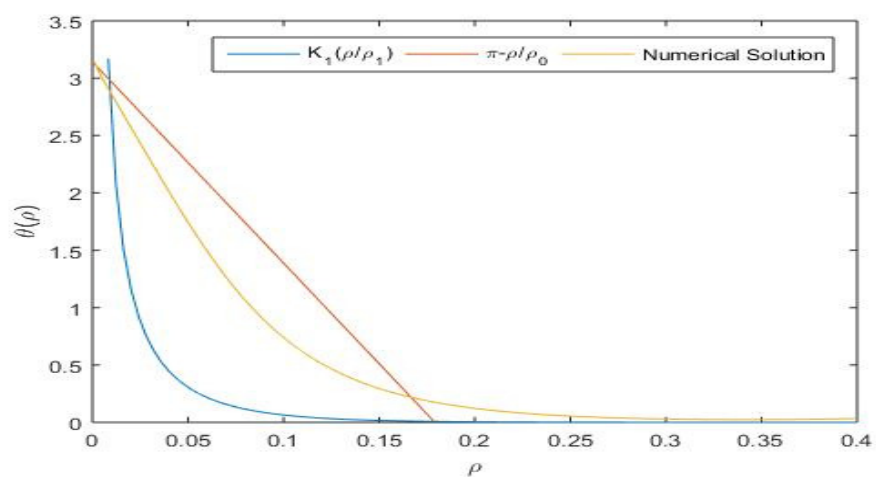

Figure 2: Comparison between the numerical solution of $(2.5)$ and the analytical linear approximations for $\frac{E}{E_{0}}=1.5$
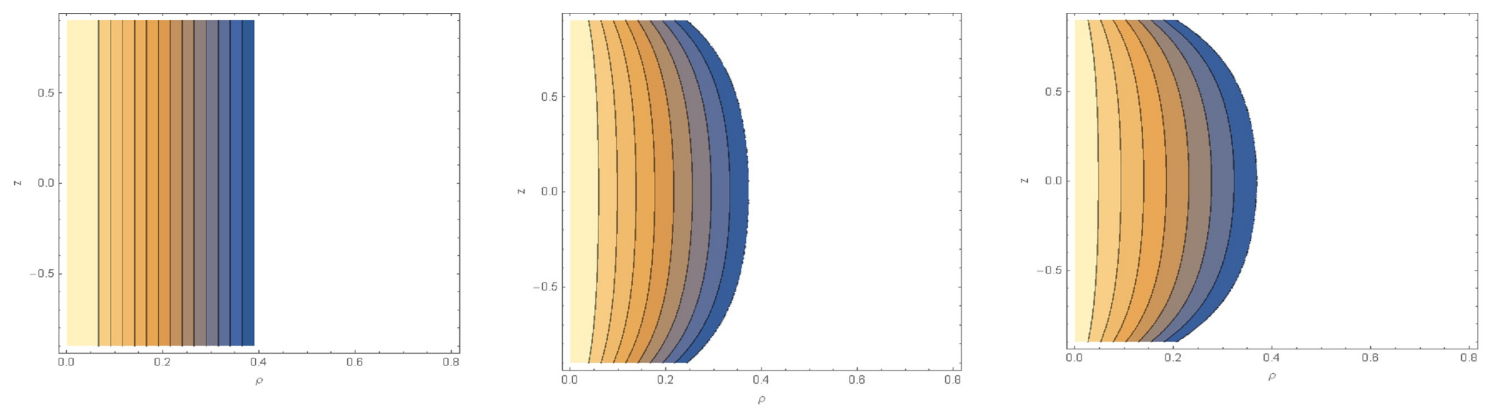

(a) Contour plot of solution 2.10 for (b) Contour plot of solution 2.10 for (c) Contour plot of solution 2.10 for $\left(E / E_{0}, \nu, k_{s}\right)=(1.02,1.8,0) . \quad\left(E / E_{0}, \nu, k_{s}\right)=(1.02,1.8,1.5) . \quad\left(E / E_{0}, \nu, k_{s}\right)=(1.02,1.8,5)$.

Figure 3: Contour plot of solution 2.10 for three different values of $k_{s}$ with constant external electric field and cell thickness.

A different representation of the spherulite is given by reporting the intersection point with the $\rho$ axis by the tangent to the inflection point of $\theta(\rho)$, for a fixed value of $z$ 10. The results of this procedure are reported in figures 6 a and $6 . \mathrm{b}$, for the two different values of $\frac{E}{E_{0}}$ taken into consideration. We stress that for greater external fields the size of all vortices narrows.

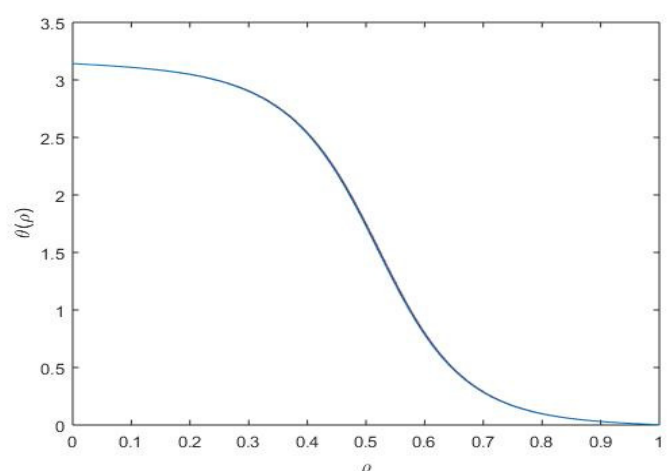

(a) $\left(\frac{E}{E_{0}}\right)=1.02, k_{s}=0.1$

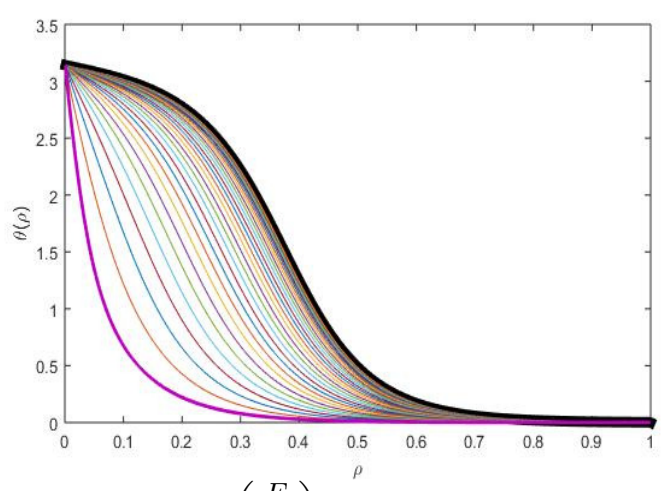

(b) $\left(\frac{E}{E_{0}}\right)=1.02, k_{s}=6$

Figure 4: Profiles $\theta(\rho)$ for $E / E_{0}=1.02$. Different curves refer to different values of $|z|$. Bold curves have to be referred to $z=0$ (the black one) and to $|z|=\nu / 2$ (the purple one). 


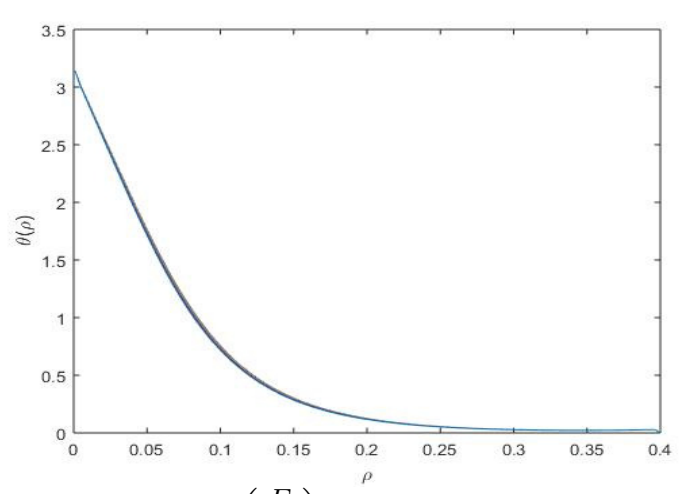

(a) $\left(\frac{E}{E_{0}}\right)=1.5, k_{s}=0.1$

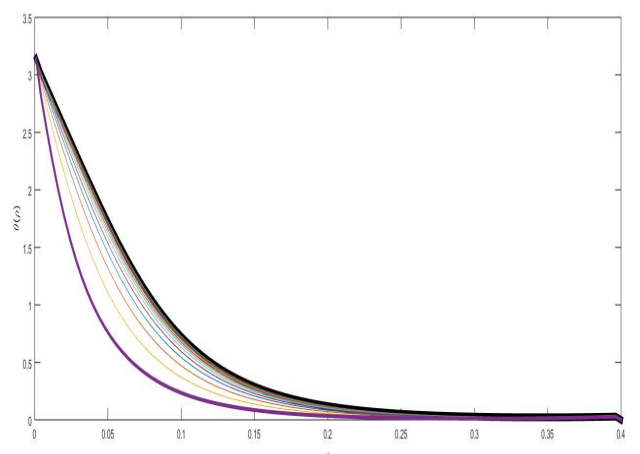

(b) $\left(\frac{E}{E_{0}}\right)=1.5, k_{s}=6$

Figure 5: Profiles $\theta(\rho)$ for $E / E_{0}=1.5$. Different curves refer to different values of $|z|$. Bold curves have to be referred to $z=0$ (the black one) and to $\|z\|=\nu / 2$ (the purple one). We note that the effect of a greater external electric field is to narrow the size of the vortices, for fixed values of $k_{s}$.

\section{Diffusion of Light on a CLC cylindrical structure}

In this section we consider the scattering of an e.m. wave, propagating through a confined CLC, which is under the suitable conditions for a spherulite to be formed. The geometry is the same as in the previous section, as the same is the choice of the cartesian axes. We assume that the wave vector is parallel to the $(x, y)$ - plane. The propagation of the wave is described in terms of the oscillating electric field $\vec{E}$, eventually to be distinguished by the static electric field $\mathbf{E}$, and by the associated electric displacement field $\vec{D}$, by the equation 35

$$
\nabla(\nabla \cdot \vec{E})-\nabla^{2} \vec{E}=-\partial_{t t} \vec{D}
$$

This equation has been obtained, as usual, by eliminating the magnetic field from the Maxwell's equations. The electric anisotropy of the CLC is made explicit by the existence of a permeability tensor, which locally has an orthogonal component $\epsilon_{\perp}$ if $\vec{E} \perp \mathbf{n}$ and by a parallel contribution $\epsilon_{\|}$if $\vec{E} \| \mathbf{n}$. Then the costitutive relation is given by $16,17,36$

$$
\vec{D}=\epsilon_{\perp} \vec{E}+\Delta \epsilon \mathbf{n}(\vec{E} \cdot \mathbf{n}), \quad \Delta \epsilon=\epsilon_{\|}-\epsilon_{\perp} .
$$

Let us assume that the incident wave is described by the electric field

$$
\vec{E} \rightsquigarrow E_{y} e^{\imath(k x-\omega t)} \mathbf{j}+e^{\imath(\tilde{k} x-\omega t)} \mathbf{k}=\overrightarrow{\mathcal{E}}_{\infty} e^{-\imath \omega t} \quad x \rightarrow-\infty,
$$

where $\tilde{k}=k \sqrt{1+\frac{\Delta \epsilon}{\epsilon_{\perp}}}$.

Since we suppose that the spherulite is not perturbed by the wave, we need to assume certain supplementary conditions:

1. the liquid crystal molecules are not deformed/rotated by wave, which implies $\omega \gg \frac{1}{\tau}$, being $\tau$ any "relaxation time" of the CLC.

2. The diffractive effects in the light scattering on the spherulite are not negligible, then we assume that its wavelength is $\lambda \lesssim \rho_{0}\left(\right.$ or $\left.k(\omega) \gtrsim \frac{1}{\rho_{0}}\right)$, being $\rho_{0}$ the typical size of the spherulite defined in equation 2.8.

3. the horizontal bounding plates are considered as homogeneous dielectric planes, thus restricting the electric field to be periodic along the $z$ axis.

4. A strong supplementary condition we introduce is $\nabla \cdot \vec{E}=0$, which may imply $\nabla \cdot \vec{D}=\rho_{\text {free }} \neq 0$. This should be true in the core of the spherulites, where we expect significant variations of the fields. However, at this stage of our analysis we prefer to adopt such an assumption, because the equations become simpler. Then an a posteriori evaluation of the local free charge density will clarify how good is our hypothesis. 


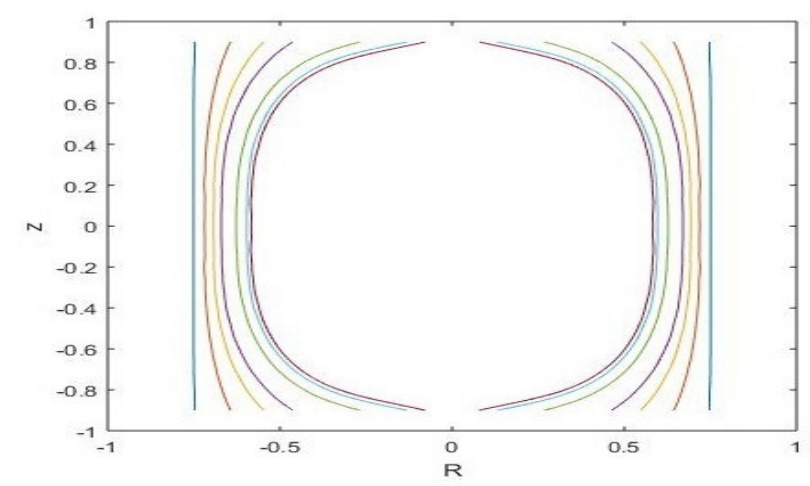

(a) $\left(\frac{E}{E_{0}}\right)=1.02$

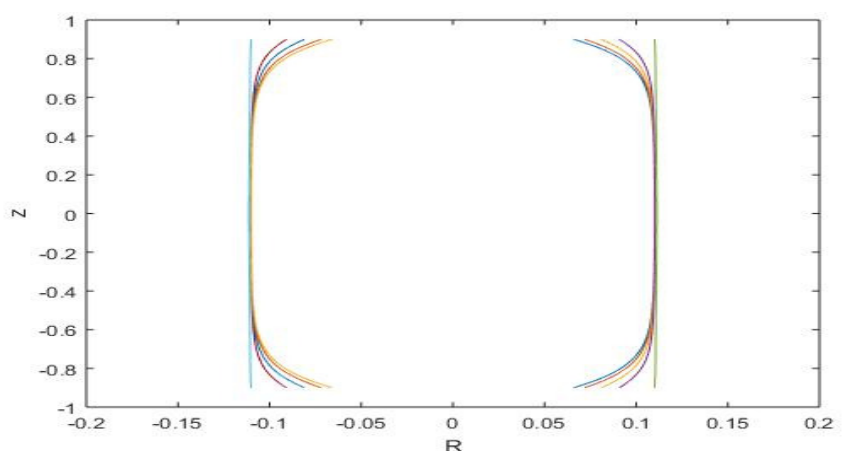

(b) $\left(\frac{E}{E_{0}}\right)^{\mathrm{R}}=1.5$

Figure 6: Size of the planar vortices for different values of $|z|$. Different colors refer to different values of $k_{s}\left(k_{s}=0.1,0.5,1,1.5,3,6,12\right)$. We note that for smaller $k_{s}$ more similar to a cylinder are the solutions. The structures tend to take the form of a bubble as $k_{s}$ increases. 
5. A final remark concerns the functional dependency of the shape of the spherulite, which we assume to be simply $\theta=\theta(\rho)$. Thus, for sake of simplicity we neglect the modulation along the $z$ axis described by 2.12.

Under the conditions above equation 3.1) leads the equation for $\overrightarrow{\mathcal{E}}=\overrightarrow{\mathcal{E}}(\vec{r})$

$$
\nabla^{2} \overrightarrow{\mathcal{E}}=-k^{2} \mathcal{A} \overrightarrow{\mathcal{E}}, \quad \overrightarrow{\mathcal{E}} \rightsquigarrow \overrightarrow{\mathcal{E}}_{\infty} \text { as } x \rightarrow-\infty
$$

where $k=\frac{\omega}{c} \sqrt{\epsilon_{\perp}}$ and the coupling matrix

$$
\mathcal{A}=\mathbf{1}_{3}+\frac{\Delta \epsilon}{\epsilon_{\perp}} \mathbf{n} \otimes \mathbf{n}
$$

Since $\mathbf{n}=\mathbf{n}(\rho, \phi)=\cos \theta(\rho) \mathbf{k}+\sin \theta(\rho) \phi$, we are naturally led to express also the electric field as

$$
\overrightarrow{\mathcal{E}}=\mathcal{E}_{\rho}(\rho, \phi, z) \boldsymbol{\rho}+\mathcal{E}_{\phi}(\rho, \phi, z) \phi+\mathcal{E}_{z}(\rho, \phi, z) \mathbf{k} .
$$

Then, now it is easier to explicit the off diagonal contributions to the 3.4. Indeed, provided that

$$
(\mathbf{n} \cdot \overrightarrow{\mathcal{E}}) \mathbf{n}=\left(\sin \theta \mathcal{E}_{\phi}+\cos \theta \mathcal{E}_{z}\right)(\cos \theta \mathbf{k}+\sin \theta \boldsymbol{\phi}),
$$

then (3.4) reads

$$
\begin{gathered}
\left(\nabla^{2}+k^{2}\right)\left(\mathcal{E}_{\rho} \boldsymbol{\rho}+\mathcal{E}_{\phi} \boldsymbol{\phi}+\mathcal{E}_{z} \mathbf{k}\right)= \\
-k^{2} \frac{\Delta \epsilon}{\epsilon_{\perp}}\left[\left(\sin \theta \mathcal{E}_{\phi}+\cos \theta \mathcal{E}_{z}\right) \cos \theta \mathbf{k}+\left(\sin \theta \mathcal{E}_{\phi}+\cos \theta \mathcal{E}_{z}\right) \sin \theta \boldsymbol{\phi}\right] .
\end{gathered}
$$

However, now the Laplacian operator acts on the cylindrical components of a vector-field, then it takes different expressions according to the component index. In particular, by defining $\nabla_{0}^{2} \cdot=\frac{1}{\rho} \partial_{\rho}\left(\rho \partial_{\rho} \cdot\right)+$ $\frac{1}{\rho^{2}} \partial_{\phi}^{2}+\partial_{z}^{2}$, equation 3.8 becomes

$$
\begin{aligned}
\left(\nabla_{0}^{2}+k^{2}\right) \mathcal{E}_{\rho} & =\frac{1}{\rho^{2}}\left(\mathcal{E}_{\rho}+2 \partial_{\phi} \mathcal{E}_{\phi}\right), \\
\left(\nabla_{0}^{2}+k^{2}\right) \mathcal{E}_{\phi} & =\frac{1}{\rho^{2}}\left(\mathcal{E}_{\phi}-2 \partial_{\phi} \mathcal{E}_{\rho}\right)-k^{2} \frac{\Delta \epsilon}{\epsilon_{\perp}}\left(\sin ^{2} \theta \mathcal{E}_{\phi}+\frac{1}{2} \sin 2 \theta \mathcal{E}_{z}\right), \\
\left(\nabla_{0}^{2}+\tilde{k}^{2}\right) \mathcal{E}_{z} & =-k^{2} \frac{\Delta \epsilon}{\epsilon_{\perp}}\left(\frac{1}{2} \sin 2 \theta \mathcal{E}_{\phi}-\sin ^{2} \theta \mathcal{E}_{z}\right), \quad \tilde{k}=k \sqrt{1+\frac{\Delta \epsilon}{\epsilon_{\perp}}} .
\end{aligned}
$$

In order to describe the scattering of the light on the spherulite, the above equations have to be solved with the asymptotic conditions

$$
\mathcal{E}_{\rho} \rightsquigarrow \mathcal{E}_{\infty \rho} \sin \phi e^{\imath k \rho \cos \phi}, \mathcal{E}_{\phi} \rightsquigarrow \mathcal{E}_{\infty \phi} \cos \phi e^{\imath k \rho \cos \phi}, \mathcal{E}_{z} \rightsquigarrow \mathcal{E}_{\infty z} e^{i \tilde{k} \rho \cos \phi} \quad \text { for } \phi \rightarrow \pm \pi \text { and } \rho \rightarrow \infty \text {. }
$$

Of course such asymptotic conditions are exact solutions of the homogeneous system above, i.e. when $\frac{\Delta \epsilon}{\epsilon} \rightarrow 0$. As the problem of finding a complete analytical solution to (3.10) - 3.11) is quite hard, let us consider a perturbative setting. The basic idea is to first give a Born approximated solution of the equation (3.11), keeping an implicit dependence on $\mathcal{E}_{\phi}$. Then we can use it in 3.10 which will become a closed linear equation, even if non local, in $\mathcal{E}_{\phi}$. Solving it, in the same approximation, one can use these results into 3.9 for $\mathcal{E}_{\rho}$.

\section{Perturbative solutions of the light scattering equations by a spherulite}

\subsection{The out plane conversion}

Following the standard method by Lippmann-Schwinger 37, let us rewrite equation (3.11) as the integral equation

$$
\mathcal{E}_{z}(\vec{r})=\mathcal{E}_{\infty z} e^{i \tilde{\imath} \rho \cos \phi}+\int G\left(\vec{r}, \vec{r}^{\prime}\right) U\left[\mathcal{E}_{z}\left(\vec{r}^{\prime}\right), \mathcal{E}_{\phi}\left(\vec{r}^{\prime}\right), \theta\left(\rho^{\prime}\right)\right] d \vec{r}^{\prime}
$$


where $U\left[\mathcal{E}_{z}(\vec{r}), \mathcal{E}_{\phi}(\vec{r}), \theta(\rho)\right]=-k^{2} \frac{\Delta \epsilon}{\epsilon_{\perp}}\left(\frac{1}{2} \sin 2 \theta(\rho) \mathcal{E}_{\phi}(\vec{r})-\sin ^{2} \theta(\rho) \mathcal{E}_{z}(\vec{r})\right)$ and the Green function $G\left(\vec{r}, \vec{r}^{\prime}\right)$ is a solution of the PDE

$$
\left(\nabla_{0}^{2}+\tilde{k}^{2}\right) G\left(\vec{r}, \vec{r}^{\prime}\right)=\frac{1}{\rho^{\prime}} \delta\left(\rho-\rho^{\prime}\right) \delta\left(\phi-\phi^{\prime}\right) \delta\left(z-z^{\prime}\right)
$$

provided that it is differentiable in its domain (i.e. the CLC layer) except at the point $\rho=\rho^{\prime}, \phi=$ $\phi^{\prime}, z=z^{\prime}$. There the partial first derivatives exist, but they are not continuous, in such a way that the second derivatives admit the singularity defined by the r.h.s. in 4.2.

The function $G$ can take the form

$$
G=\frac{1}{2 \pi \nu} \sum_{m, n=-\infty}^{\infty} e^{\frac{2 \pi \imath n}{\nu}\left(z-z^{\prime}\right)} e^{\imath m\left(\phi-\phi^{\prime}\right)} h_{m, n}\left(\rho, \rho^{\prime}\right),
$$

where the functions $h_{n, m}$ satisfy the Bessel type equation with singular inhomogeneity 32

$$
\left[\frac{1}{\rho} \partial_{\rho}\left(\rho \partial_{\rho} \cdot\right)-\frac{m^{2}}{\rho^{2}}+\tilde{k}^{2}-\left(\frac{2 \pi n}{\nu}\right)^{2}\right] h_{m, n}=\frac{1}{\rho^{\prime}} \delta\left(\rho-\rho^{\prime}\right),
$$

and the cutoff frequency $\kappa_{n}=\sqrt{\tilde{k}^{2}-\left(\frac{2 \pi n}{\nu}\right)^{2}}$ for $\tilde{k} \geq \frac{2 \pi n}{\nu}$. is induced by the finite transverse size of the CLC layer.

We require $G$ to be a continuous function with a bounded behaviour at $\rho \rightarrow 0$ and, additionally, to be a cylindrical progressive wave as $\rho \rightarrow \infty$, i.e. of the form $\propto \frac{e^{2 \kappa \rho}}{\sqrt{\kappa \rho}}$. Furthemore, because of the $\delta$ like inhomogeneity, $G$ can have discontinuities only in the first derivatives at $\rho \rightarrow \rho^{\prime}$.

In conclusion, by imposing the above conditions, the Green function (4.3) takes the form

$$
G\left(\vec{r}, \vec{r}^{\prime}\right)=\frac{-\imath}{4 \nu} \sum_{m, n=-\infty}^{\infty} e^{\frac{2 \pi \imath n}{\nu}\left(z-z^{\prime}\right)} e^{\imath m\left(\phi-\phi^{\prime}\right)} H_{m}^{(1)}\left(\kappa_{n} \rho_{>}\right) J_{m}\left(\kappa_{n} \rho_{<}\right), \quad\left\{\begin{array}{l}
\rho_{>}=\max \left(\rho, \rho^{\prime}\right) \\
\rho_{<}=\min \left(\rho, \rho^{\prime}\right)
\end{array}\right.
$$

where $J_{m}$ denotes the Bessel function of first kind with integer order $m$ and $H_{m}^{(1)}(\zeta)=J_{m}(\zeta)+\imath Y_{m}(\zeta)$ the corresponding Hankel function of first kind [32. Without further calculations, dramatic simplifications stem from our assumption 5. in Sec. 3, implying that the only non vanishing contributions come from the $n=0$ mode. Moreover, we are actually interested in the behaviour of the wave at radii much larger than the effective size of the spherulite, which decreases very fast, as we noticed in (2.14). Thus the form of Green function we have to use is

$$
G_{\text {sempl }}\left(\vec{r}, \vec{r}^{\prime}\right)=\frac{-\imath}{4} \sum_{m=-\infty}^{\infty} e^{\imath m\left(\phi-\phi^{\prime}\right)} H_{m}^{(1)}(\tilde{k} \rho) J_{m}\left(\tilde{k} \rho^{\prime}\right),
$$

Now, replacing the above formula into (4.1) and introducing the explicit form of the potential $U$, we see from (3.11) that the parameter $\frac{\Delta \epsilon}{\epsilon_{\perp}}$ can be considered as a perturbation parameter, allowing to express the wave function as a series of powers of it. At the 0 order the solution is given by asymptotics (3.12), which replaced into (4.1) provides at the first order (Born approximation) corrections to the plane wave propagation.

Thus, in the Born limit, by the identity $e^{\imath \zeta \cos p}=\sum_{l=-\infty}^{\infty} e^{\frac{2 \pi l}{2}} e^{\imath l p} J_{l}(\zeta)$, one can integrate on $\phi^{\prime}$ and obtain the approximated expression $\mathcal{E}_{z}^{B}(\vec{r})$ of the $\mathcal{E}_{z}(\vec{r})$ component as follows

$$
\begin{gathered}
\mathcal{E}_{z}^{B}(\vec{r})=\mathcal{E}_{\infty z} e^{i \tilde{k} \rho \cos \phi}+\frac{\pi}{2} \frac{\Delta \epsilon k^{2}}{\epsilon \perp} \sum_{m=-\infty}^{\infty} e^{i m \phi} H_{m}^{(1)}(\tilde{k} \rho) \\
\int\left[\mathcal{E}_{\infty \phi} \frac{\imath^{m}}{4} \sin \left(2 \theta\left(\rho^{\prime}\right)\right) J_{m}\left(\tilde{k} \rho^{\prime}\right)\left(J_{m-1}\left(k \rho^{\prime}\right)-J_{m+1}\left(k \rho^{\prime}\right)\right)-\mathcal{E}_{\infty \mathrm{Z}} \imath^{m+1} \sin ^{2}\left(\theta\left(\rho^{\prime}\right)\right) J_{m}^{2}\left(\tilde{k} \rho^{\prime}\right)\right] \rho^{\prime} d \rho^{\prime} .
\end{gathered}
$$

In order to have a simple estimation of the integrals in the above expression, let us resort to the asymptotic expressions of the spherulite given by (2.9) and (2.14). Actually, the simplest rough choice is 2.10 (with $Z(z)=1$ ), which we will adopt here, since we are not interested in the exact values of the 


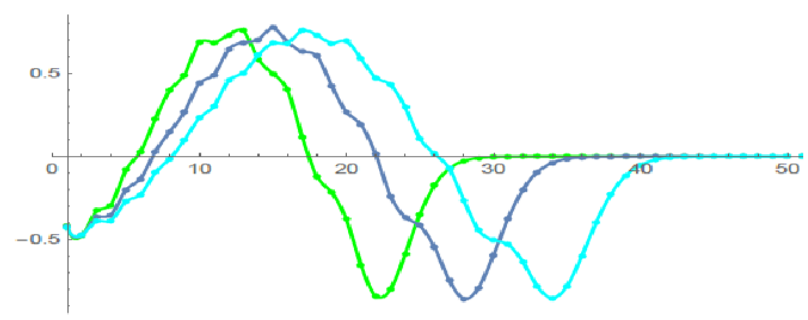

Figure 7: The numerical values of $\mathcal{I}_{m}^{\phi}$ as function of $0 \leq m \leq 50$ for three different values of $k \rho_{0}$, precisely 8 (green), 10 (blue) and 12 (cyan). They can be approximated by continuous functions in $m$, for instance by linear combinations of gaussian functions, but still a clear pattern for a systematic approximation has to be developed. It is clear a linear dependence, with a coefficient $\sim 4$, of the number of significantly terms with the size parameter $k \rho_{0}$. This is in agreement with the scattering features on localized central potentials.

diffusion amplitudes, but only in their approximate size. Thus, we have to evaluate integrals of the form

$$
\begin{aligned}
\mathcal{I}_{m}^{\phi}= & -\int_{0}^{\pi k \rho_{0}} \sin \left(2 \frac{s}{k \rho_{0}}\right) J_{m}(s)\left(J_{m-1}(s)-J_{m+1}(s)\right) s d s= \\
& -\int_{0}^{\pi k \rho_{0}} \sin \left(2 \frac{s}{k \rho_{0}}\right)\left(J_{m}(s)^{2}\right)^{\prime} s d s, \\
\mathcal{I}_{m}^{z}= & \int_{0}^{\pi k \rho_{0}} \sin ^{2}\left(\frac{s}{k \rho_{0}}\right) J_{m}^{2}(s) s d s,
\end{aligned}
$$

where the substitution $\tilde{k} \rightarrow k$ is justified, since the difference is of the order $\frac{\Delta \epsilon}{\epsilon_{\perp}}$ as stated in (3.11).

At the moment the above matrix elements do not have yet an analytical expression and should be computed numerically.

Examples of the numerical evaluation of a certain number of integrals 4.8 is given in figure 7 .

Before proceeding in such a calculations let us show the form of the cross section of conversion of a in plane polarized wave into a out plane polarized one. In fact let us suppose $\mathcal{E}_{\infty z}=0$, then the scattered amplitude along the $z$-axis 4.7 reads

$$
\mathcal{E}_{z}^{B}(\vec{r})=\mathcal{E}_{\infty \phi} \frac{\pi \Delta \epsilon}{8 \epsilon_{\perp}} \sum_{m=-\infty}^{\infty}{ }^{m} \mathcal{I}_{m}^{\phi} e^{i m \phi} H_{m}^{(1)}(\tilde{k} \rho) .
$$

Recalling that at infinity the asymptotic behaviour of the Hankel functions is $H_{m}^{(1)}(\zeta) \rightsquigarrow \frac{(1-i) e^{i \zeta-\frac{i \pi m}{2}}}{\sqrt{\pi \zeta}}+$ $O\left(\zeta^{-3 / 2}\right)$, the above expression becomes

$$
\begin{aligned}
\mathcal{E}_{z}^{B}(\vec{r})= & (1-\imath) \mathcal{E}_{\infty \phi} \frac{\pi \Delta \epsilon}{8 \epsilon \perp} \frac{e^{\imath \tilde{k} \rho}}{\sqrt{\pi \tilde{k} \rho}} \sum_{m=-\infty}^{\infty} \mathcal{I}_{m}^{\phi} e^{i m \phi}= \\
& (1-\imath) \mathcal{E}_{\infty \phi} \frac{\pi \Delta \epsilon}{8 \epsilon_{\perp}} \frac{e^{\imath \tilde{k} \rho}}{\sqrt{\pi \tilde{k} \rho}}\left[\mathcal{I}_{0}^{\phi}+2 \sum_{m=1}^{+\infty} \mathcal{I}_{m}^{\phi} \cos m \phi\right],
\end{aligned}
$$

where the identity $\mathcal{I}_{-m}^{\phi}=\mathcal{I}_{m}^{\phi}$ has been used, which can be easily proved from 4.8 and by $J_{-m}=$ $(-1)^{m} J_{m}$. 
The cross section of the conversion of linear in plane polarized $\hat{y}$ light into the out plane $\hat{z}$ one is given by

$$
\frac{d \sigma_{c o n v}}{d \phi}(\hat{r}, \hat{z} ; \hat{x}, \hat{y})=\frac{\pi}{32} \sqrt{\frac{\epsilon_{\perp}}{\epsilon_{\|}}}\left(\frac{\Delta \epsilon}{\epsilon_{\perp}}\right)^{2} \frac{\nu \rho_{0}}{k \rho_{0}}\left[\mathcal{I}_{0}^{\phi}+2 \sum_{m=1}^{+\infty} \mathcal{I}_{m}^{\phi} \cos m \phi\right]^{2},
$$

where we have singled out the dependency on the geometrical size of the spherulites from its relative size with respect the used light wavelength.

The calculations of the conversion cross section in the direction $\hat{r}(\phi)$ indicates that there is a quite well defined small angle, around $10^{\circ}$ in our numerical examples, along which the rotation of the polarization is efficiently performed. The angle of maximum conversion is $\propto\left(k \rho_{0}\right)^{-1}$, thus it becomes smaller as the wavelength becomes shorter. A further remarkable aspect is the vanishing of the backscattering. The effective values depend basically on the square of the anisotropy ratio $\left(\frac{\Delta \epsilon}{\epsilon_{\perp}}\right)^{2}$. In fact the total cross section takes the expression

$$
\sigma_{\text {conv }}=\frac{\pi^{2}}{16} \sqrt{\frac{\epsilon_{\perp}}{\epsilon_{\|}}}\left(\frac{\Delta \epsilon}{\epsilon_{\perp}}\right)^{2} \frac{\nu \rho_{0}}{k \rho_{0}}\left[\left(\mathcal{I}_{0}^{\phi}\right)^{2}+2 \sum_{m=1}^{\infty}\left(\mathcal{I}_{m}^{\phi}\right)^{2}\right]
$$

which is a decreasing function of $k \rho_{0}$.

Let us turn our attention again to equation 4.7). By using the exact numerical solutions for the spherulite (figures 4 and 5), we can obtain the exact expression of the differential cross section in (4.11), for the scattering of an electromagnetic wave by a skyrmion. A direct comparison between the exact differential cross section and the approximated one in arbitrary units is reported in figures 9 and 10 . As it can be seen, the exact numerical solution for the spherulites makes the angle of maximum conversion smaller, than the one computed through the use of the approximated solution. Furthermore, recalling that as the external electric field increases, the size of the spherulite decreases (as described by equation (2.8), we note that the larger is the size of the skyrmion, more efficient is the polarization conversion with respect to the approximated one.

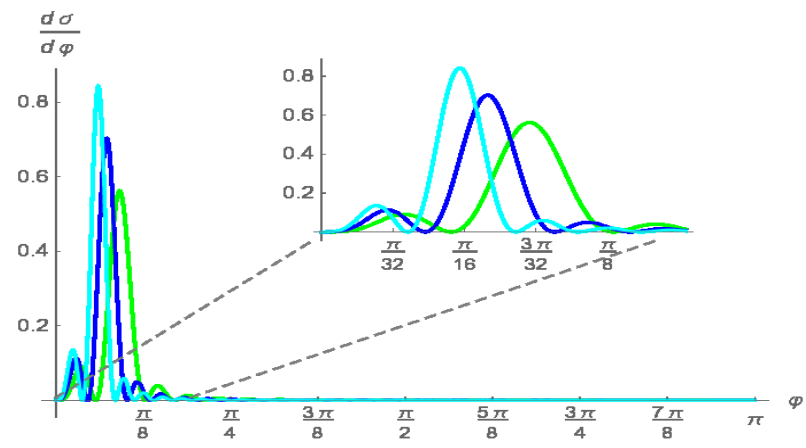

Figure 8: The numerical evaluation of the conversion cross section (4.11), in arbitrary units, for the three different values of $\tilde{k} \rho_{0}=8,10,12$, by using the same color code as in fig. (7).

\subsection{The in plane conversion}

Now let us turn our attention on the subsystem 3.9 - 3.10, which could be represented in the form

$$
\left(\begin{array}{cc}
L+k^{2} & -M \\
M & L+k^{2}
\end{array}\right)\left(\begin{array}{c}
\mathcal{E}_{\rho} \\
\mathcal{E}_{\phi}
\end{array}\right)=k^{2} \frac{\Delta \epsilon}{\epsilon_{\perp}}\left(\begin{array}{c}
0 \\
\sin ^{2} \theta \mathcal{E}_{\phi}+\frac{1}{2} \sin 2 \theta \mathcal{E}_{z}
\end{array}\right),
$$




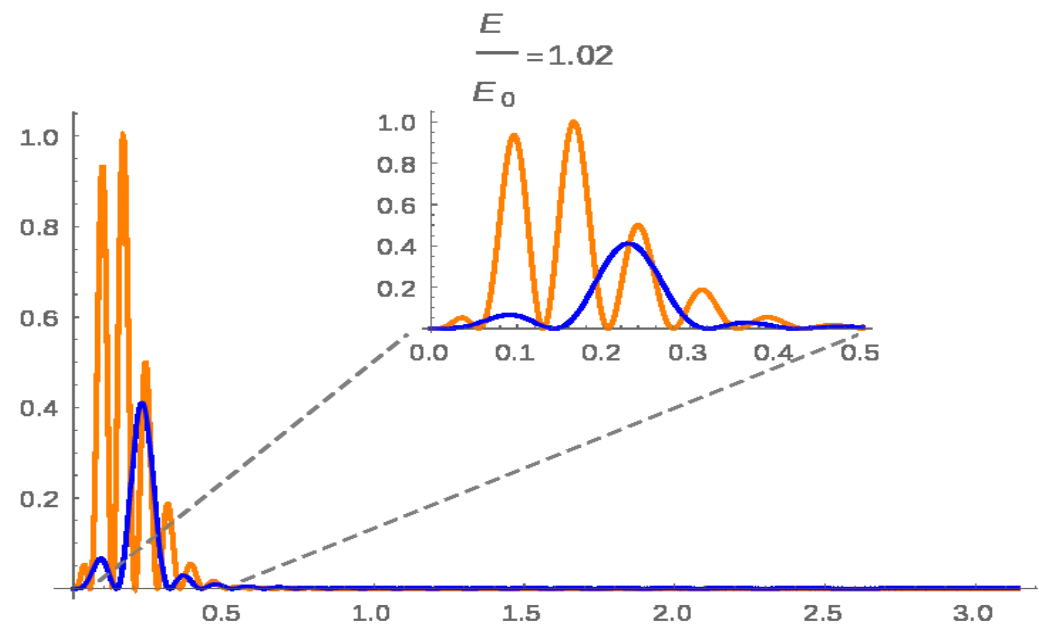

Figure 9: The numerical evaluation of the conversion cross section 4.11), in arbitrary units, for $\rho_{0}$ fixed by $(2.8)$ and the ratio $E / E_{0}=1.02, k \rho_{0}=10$ and for the exact numerical solution (orange) and the approximated solution 2.9 (blue).

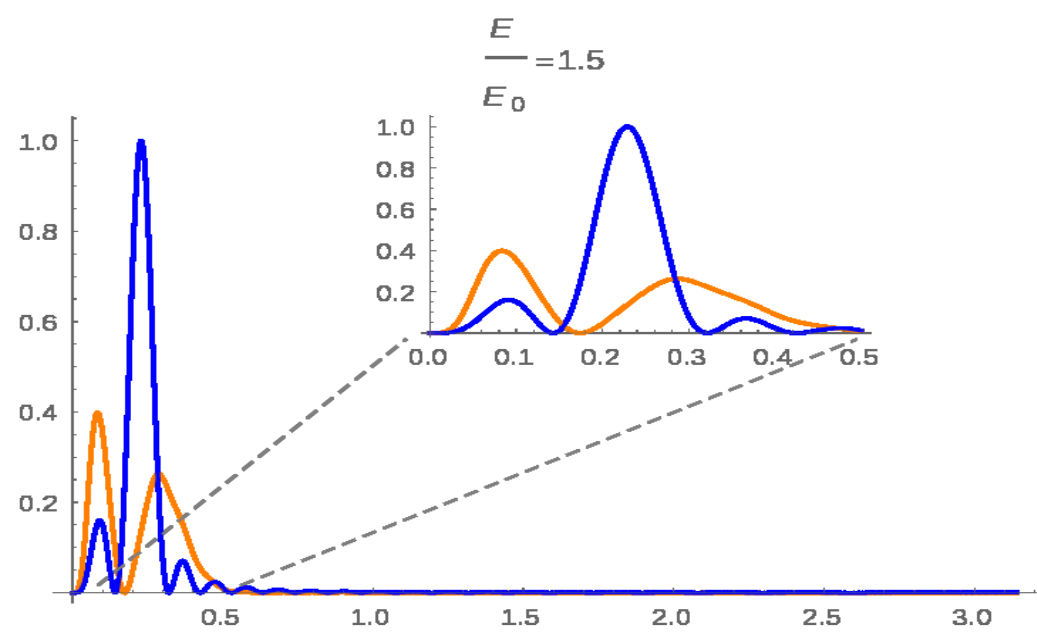

Figure 10: The numerical evaluation of the conversion cross section (4.11), in arbitrary units, for $\rho_{0}$ fixed by (2.8) and the ratio $E / E_{0}=1.5, k \rho_{0}=10$ and for the exact numerical solution (orange) and the approximated solution 2.9 (blue). 
where $L=\nabla_{0}^{2}-\frac{1}{\rho^{2}}$ and $M=\frac{2}{\rho^{2}} \partial_{\phi}$. As before, it can be set into the integral form

$$
\begin{aligned}
\left(\begin{array}{c}
\mathcal{E}_{\rho} \\
\mathcal{E}_{\phi}
\end{array}\right) & =\left(\begin{array}{c}
\mathcal{E}_{\infty \rho} \sin \phi \\
\mathcal{E}_{\infty \phi} \cos \phi
\end{array}\right) e^{\imath k \rho \cos \phi}+ \\
k^{2} \frac{\Delta \epsilon}{\epsilon_{\perp}} & \int\left(\begin{array}{cc}
G\left(\vec{r}, \vec{r}^{\prime}\right) & -F\left(\vec{r}, \vec{r}^{\prime}\right) \\
F\left(\vec{r}, \vec{r}^{\prime}\right) & G\left(\vec{r}, \vec{r}^{\prime}\right)
\end{array}\right)\left(\begin{array}{c}
0 \\
\sin ^{2} \theta\left(\vec{r}^{\prime}\right) \mathcal{E}_{\phi}\left(\vec{r}^{\prime}\right)+\frac{1}{2} \sin 2 \theta\left(\vec{r}^{\prime}\right) \mathcal{E}_{z}\left(\vec{r}^{\prime}\right)
\end{array}\right) d \vec{r}^{\prime} .
\end{aligned}
$$

Of course, the inhomogeneous term is a solution of the homogeneous system (4.13) and the matrix Green function is

$$
\left(\begin{array}{cc}
G\left(\vec{r}, \vec{r}^{\prime}\right) & -F\left(\vec{r}, \vec{r}^{\prime}\right) \\
F\left(\vec{r}, \vec{r}^{\prime}\right) & G\left(\vec{r}, \vec{r}^{\prime}\right)
\end{array}\right)=\frac{1}{2 \pi \nu} \sum_{m, n=-\infty}^{\infty} e^{\frac{2 \pi \imath n}{\nu}\left(z-z^{\prime}\right)} e^{\imath m\left(\phi-\phi^{\prime}\right)}\left(\begin{array}{cc}
h_{m, n}\left(\rho, \rho^{\prime}\right) & -\imath f_{m, n}\left(\rho, \rho^{\prime}\right) \\
\imath f_{m, n}\left(\rho, \rho^{\prime}\right) & h_{m, n}\left(\rho, \rho^{\prime}\right)
\end{array}\right)
$$

where the unknown $h_{m, n}, f_{m, n}$ satisfy the matrix equation

$$
\left(\begin{array}{cc}
\frac{1}{\rho} \partial_{\rho}\left(\rho \partial_{\rho} \cdot\right)-\frac{m^{2}+1}{\rho^{2}}+\kappa_{n}^{2} & \frac{-2 \imath m}{\rho^{2}} \\
\frac{2 \imath m}{\rho^{2}} & \frac{1}{\rho} \partial_{\rho}\left(\rho \partial_{\rho} \cdot\right)-\frac{m^{2}+1}{\rho^{2}}+\kappa_{n}^{2}
\end{array}\right)\left(\begin{array}{cc}
h_{m, n}\left(\rho, \rho^{\prime}\right) & -\imath f_{m, n}\left(\rho, \rho^{\prime}\right) \\
\imath f_{m, n}\left(\rho, \rho^{\prime}\right) & h_{m, n}\left(\rho, \rho^{\prime}\right)
\end{array}\right)=\frac{\mathbf{1}_{2}}{\rho^{\prime}} \delta\left(\rho-\rho^{\prime}\right),
$$

where $\kappa_{n}^{2}=k^{2}-\left(\frac{2 \pi n}{\nu}\right)^{2}$.

As in the previous subsection, we limit ourselves to evaluate the diffusion of light by the spherulite in the Born approximation. Accordingly, the conversion from out-plane to in-plane scattering leads to the following approximated expression

$$
\left(\begin{array}{c}
\mathcal{E}_{\rho}^{B} \\
\mathcal{E}_{\phi}^{B}
\end{array}\right)=\mathcal{E}_{\infty z} \frac{k^{2}}{2 \pi \nu} \frac{\Delta \epsilon}{2 \epsilon_{\perp}} \sum_{m, n=-\infty}^{\infty} \int \sin 2 \theta\left(\rho^{\prime}\right) e^{\imath \tilde{k} \rho^{\prime} \cos \phi^{\prime}} e^{\frac{2 \pi \imath n}{\nu}\left(z-z^{\prime}\right)} e^{\imath m\left(\phi-\phi^{\prime}\right)}\left(\begin{array}{c}
-\imath f_{m, n}\left(\rho, \rho^{\prime}\right) \\
h_{m, n}\left(\rho, \rho^{\prime}\right)
\end{array}\right) d \vec{r}^{\prime}
$$

where $h_{m, n}$ and $f_{m, n}$ are solutions of the system 4.16). Again, using the simplification induced by the assumption 5. in Sec. 3 and by using the expansion of the plane wave factor in terms of Bessel functions, one gets

$$
\left(\begin{array}{c}
\mathcal{E}_{\rho}^{B} \\
\mathcal{E}_{\phi}^{B}
\end{array}\right)=\mathcal{E}_{\infty z} \frac{\Delta \epsilon k^{2}}{2 \epsilon_{\perp}} \sum_{m=-\infty}^{\infty} \imath^{m} e^{\imath m \phi} \int \sin 2 \theta\left(\rho^{\prime}\right) J_{m}\left(\tilde{k} \rho^{\prime}\right)\left(\begin{array}{c}
-\imath f_{m}\left(\rho, \rho^{\prime}\right) \\
h_{m}\left(\rho, \rho^{\prime}\right)
\end{array}\right) \rho^{\prime} d \rho^{\prime}
$$

where we dropped the subscript $n$ from both $h_{m, n}$ and $f_{m, n}$ as the only non vanishing contributions come from the $n=0$ mode. The squared modulus of the above quantity, properly managed, will produce the cross section of the out plane - in plane scattering process.

From 4.16 we obtain the equations for $h_{m}$ and $f_{m}$ as follows

$$
\left\{\begin{array}{c}
h_{m}^{\prime \prime}(\rho)+\frac{h_{m}^{\prime}(\rho)}{\rho}+\left(k^{2}-\frac{m^{2}+1}{\rho^{2}}\right) h_{m}(\rho)+\frac{2 m f_{m}(\rho)}{\rho^{2}}=\frac{\delta\left(\rho-\rho^{\prime}\right)}{\rho^{\prime}} \\
f_{m}^{\prime \prime}(\rho)+\frac{f_{m}^{\prime}(\rho)}{\rho}+\left(k^{2}-\frac{m^{2}+1}{\rho^{2}}\right) f_{m}(\rho)+\frac{2 m h_{m}(\rho)}{\rho^{2}}=0
\end{array}\right.
$$

The general solution of the system above is

$$
\begin{aligned}
h_{m}^{ \pm} & =c_{1}^{ \pm} J_{m-1}(k \rho)+\imath c_{2}^{ \pm} Y_{m-1}(k \rho)+d_{1}^{ \pm} J_{m+1}(k \rho)+\imath d_{2}^{ \pm} Y_{m+1}(k \rho), \\
f_{m}^{ \pm} & =c_{1}^{ \pm} J_{m-1}(k \rho)+\imath c_{2}^{ \pm} Y_{m-1}(k \rho)-d_{1}^{ \pm} J_{m+1}(k \rho)-\imath d_{2}^{ \pm} Y_{m+1}(k \rho),
\end{aligned}
$$

where $c_{i}^{ \pm}$and $d_{i}^{ \pm}$are four arbitrary constants in the regions $\rho<\rho^{\prime}$ or $\rho>\rho^{\prime}$, respectively. Continuity of the solutions and discontinuity of their first derivatives at $\rho^{\prime}$ imply a functional dependency of those coefficients on this variable. Moreover, as in the previous section, we require regularity at $\rho \rightarrow 0$ and radiative behaviour at $\rho \rightarrow \infty$.

All conditions above lead to a linear system, from which one obtains the values of the unknown coefficients, namely

$$
\begin{array}{r}
c_{1}^{+}=-\frac{\imath \pi}{4} H_{m-1}^{(1)}\left(k \rho^{\prime}\right), d_{1}^{+}=-\frac{\imath \pi}{4} H_{m+1}^{(1)}\left(k \rho^{\prime}\right), c_{2}^{+}=d_{2}^{+}=0 \\
c_{1}^{-}=c_{2}^{-}=-\frac{\imath \pi}{4} J_{m-1}\left(k \rho^{\prime}\right), d_{1}^{-}=d_{2}^{-}=-\frac{\imath \pi}{4} J_{m+1}\left(k \rho^{\prime}\right) .
\end{array}
$$




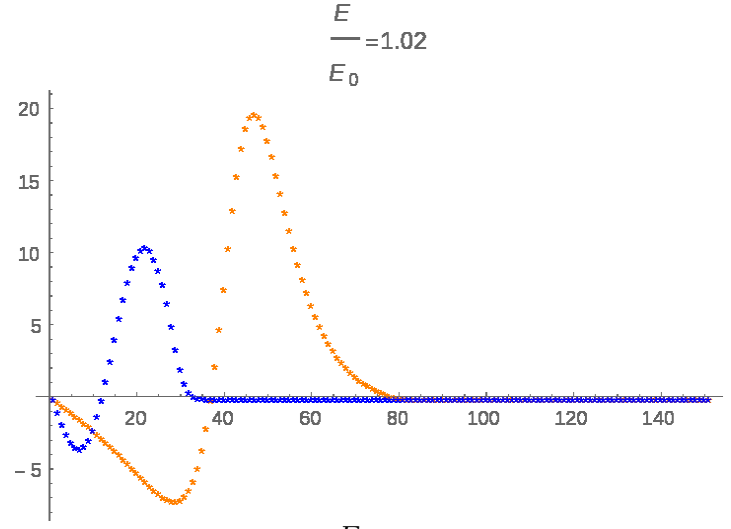

(a) $\frac{E}{E_{0}}=1.02$
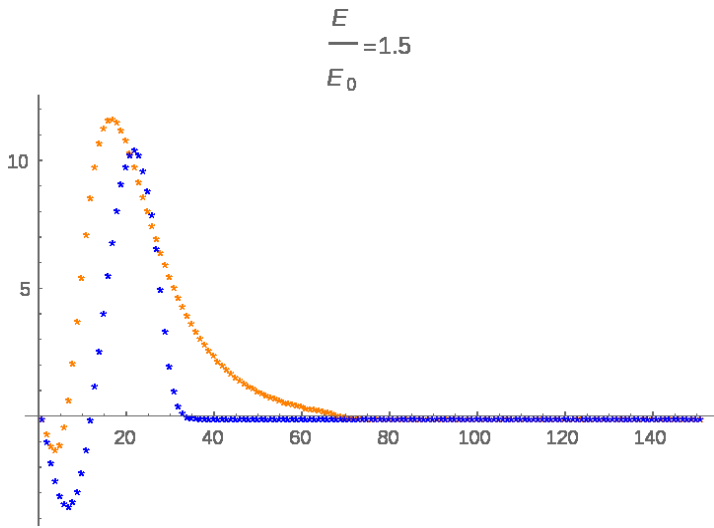

(b) $\frac{E}{E_{0}}=1.5$

Figure 11: The numerical values of $I_{m}^{(\rho)}$ as function of $0 \leq m \leq 150$, for two different values of $E / E_{0}$ with fixed $k \rho_{0}$, computed through the use of the exact numerical solution (orange) and the approximated solution (2.9) (blue).

Now we are in position to evaluate (4.18), namely

$\frac{\mathcal{E}_{\infty z} \pi \Delta \epsilon k^{2}}{8 \epsilon_{\perp}} \sum_{m=-\infty}^{\infty} \imath^{m-1} e^{\imath m \phi} \int \sin 2 \theta\left(\rho^{\prime}\right) J_{m}\left(\tilde{k} \rho^{\prime}\right)\left(\begin{array}{c}-\imath\left[J_{m-1}\left(k \rho^{\prime}\right) H_{m-1}^{(1)}(k \rho)-J_{m+1}\left(k \rho^{\prime}\right) H_{m+1}^{(1)}(k \rho)\right] \\ J_{m-1}\left(k \rho^{\prime}\right) H_{m-1}^{(1)}(k \rho)+J_{m+1}\left(k \rho^{\prime}\right) H_{m+1}^{(1)}(k \rho)\end{array}\right) \rho^{\prime} d \rho^{\prime}$.

Resorting again to the asymptotic behaviour of the Hankel functions, the solution at infinity can be estimated as

$$
\frac{\mathcal{E}_{\infty z} \sqrt{\pi} \Delta \epsilon k^{2}}{2^{\frac{5}{2}} \epsilon_{\perp}} e^{-\imath \frac{\pi}{4}} \frac{e^{\imath k \rho}}{\sqrt{k \rho}} \sum_{m=-\infty}^{\infty} e^{\imath m \phi} \int \sin 2 \theta\left(\rho^{\prime}\right) J_{m}\left(\tilde{k} \rho^{\prime}\right)\left(\begin{array}{c}
-\imath\left[J_{m-1}\left(k \rho^{\prime}\right)+J_{m+1}\left(k \rho^{\prime}\right)\right] \\
J_{m-1}\left(k \rho^{\prime}\right)-J_{m+1}\left(k \rho^{\prime}\right)
\end{array}\right) \rho^{\prime} d \rho^{\prime} .
$$

Setting

$$
\begin{aligned}
& \frac{1}{\tilde{k}^{2}} I_{m}^{(\rho)}=\frac{1}{k^{2}} \int \sin 2 \theta\left(\frac{s}{\bar{k}}\right) J_{m}(s)\left[J_{m-1}\left(k \frac{s}{\bar{k}}\right)+J_{m+1}\left(k \frac{s}{\bar{k}}\right)\right] s d s d \phi^{\prime} \\
& \frac{1}{\tilde{k}^{2}} I_{m}^{(\phi)}=\frac{1}{k^{2}} \int \sin 2 \theta\left(\frac{s}{\bar{k}}\right) J_{m}(s)\left[J_{m-1}\left(k \frac{s}{\bar{k}}\right)-J_{m+1}\left(k \frac{s}{\bar{k}}\right)\right] s d s,
\end{aligned}
$$

equation 4.23 can be rewritten as

$$
\frac{\mathcal{E}_{\infty z} \sqrt{\pi} \Delta \epsilon}{2^{\frac{5}{2}} \epsilon_{\perp}} e^{-\imath \frac{\pi}{4}} \frac{e^{\imath k \rho}}{\sqrt{k \rho}} \sum_{m=-\infty}^{\infty} e^{\imath m \phi}\left(\begin{array}{c}
-\imath I_{m}^{(\rho)} \\
I_{m}^{(\phi)}
\end{array}\right) .
$$

Recalling the identity $J_{-m}=(-1)^{m} J_{m}$, it is easy to show that $I_{0}^{(\rho)}=0, I_{m}^{(\rho)}=-I_{-m}^{(\rho)}$ and $I_{m}^{(\phi)}=I_{-m}^{(\phi)}$, so that equation 4.26 now reads

$$
\frac{\mathcal{E}_{\infty z} \sqrt{\pi} \Delta \epsilon}{2^{\frac{5}{2}} \epsilon_{\perp}} e^{-\imath \frac{\pi}{4}} \frac{e^{\imath k \rho}}{\sqrt{k \rho}}\left(\begin{array}{c}
2 \sum_{m=1}^{\infty} I_{m}^{(\rho)} \sin m \phi \\
I_{0}^{(\phi)}+2 \sum_{m=1}^{\infty} I_{m}^{(\phi)} \cos m \phi
\end{array}\right) .
$$

Performing again the substitution $\tilde{k} \rightarrow k$, we notice that $I_{m}^{(\phi)}$ is the same as $\mathcal{I}_{m}^{\phi}$ in equation 4.8). On the other hand, the values of the first three hundred matrix elements 4.24 are presented in figure 11

The in plane-conversion cross section is then given by

$$
\frac{d \sigma}{d \phi}(\hat{r}, \hat{\phi} ; \hat{x}, \hat{y})=\frac{\pi}{32} \sqrt{\frac{\epsilon_{\perp}}{\epsilon_{\|}}}\left(\frac{\Delta \epsilon}{\epsilon_{\perp}}\right)^{2} \frac{\nu \rho_{0}}{k \rho_{0}}\left[4\left(\sum_{m=1}^{\infty} I_{m}^{(\rho)} \sin m \phi\right)^{2}+\left(I_{0}^{(\phi)}+2 \sum_{m=1}^{+\infty} I_{m}^{(\phi)} \cos m \phi\right)^{2}\right]
$$

and the total cross section reads

$$
\sigma_{\text {tot }}(\hat{r}, \hat{\phi} ; \hat{x}, \hat{y})=\frac{\pi^{2}}{16} \sqrt{\frac{\epsilon_{\perp}}{\epsilon_{\|}}}\left(\frac{\Delta \epsilon}{\epsilon_{\perp}}\right)^{2} \frac{\nu \rho_{0}}{k \rho_{0}}\left[\left(I_{0}^{(\phi)}\right)^{2}+2 \sum_{m=1}^{\infty}\left(I_{m}^{(\rho)}\right)^{2}+2 \sum_{m=1}^{+\infty}\left(I_{m}^{(\phi)}\right)^{2}\right] .
$$


The numerical results, in arbitrary units, for the computation of the differential cross section (4.28) are depicted in figures 12 and 13 , for two different values of the ratio $E / E_{0}$. Conversely to what happens for the out plane-conversion cross section, in this case the use of the exact solution for the computation of the differential cross section 4.28 keeps the angle of maximum conversion substantially unchanged.

\section{Conclusions}

In the present work we showed that the spherulites in CLC can be used to change the polarization axes of incoming light with a certain efficiency. To the best of our knowledge, this phenomenon is quite new as, so far, only the light diffusion from helicoidal CLC structures in the bulk has been studied 38] : here we considered the interaction with localized perturbations, i.e. the spherulites. In detail we first described the shape of the spherulites, for different values of the controlling parameters, in particular the external applied electric (or magnetic) field. From that we were able to compute the cross section of the polarization axes conversions in Born approximation. We found that the conversion processes have maximum differential cross section at small non-zero deflection angles. Thus, the effect we described can be detected off the forward direction. Furthermore, we compared the differential cross sections for different values of the external electric field, proving that the scattering in significantly influenced by such a parameter. Thus, we can use it as a tuning controller of the diffusion. In particular, the conversion is more efficient for fields slightly above the threshold of the critical unwinding field of the cholesteric-nematic transition. This is due to the quadratic inverse dependency on the external field of the spherulite core size. In order to obtain these results, we used both a piecewise linear approximation of the spherulite profile and the corresponding numerical exact solution. On the other hand, we showed that the spherulite is badly approximated by a piecewise linear function, especially for weak electric field. Thus, to improve our results we need to further study analytical profile solution of the spherulites. Actually there are many similar questions to be answered. First, it would be important to study the cross sections for all channels, beyond the Born approximation, and to suppress the several simplifications we made. In particular, the spherulite is not a cylinder, as we assumed in the present work, but it resembles more a sort of barrel. Correspondingly, new diffractive effects may arise from the actual shape, especially close the confining plates. This is related to the type of anchoring, which is parametrised by a further controlling parameter in the Rapini-Papoular conditions. In fact, we showed that the shape of the spherulite depends significantly on it, even if the ratio $E / E_{0}$ is kept fixed. Finally, it is well known that for external fields below the critical threshold, lattice configurations of spherulites can appear 10]. This fact suggests to explore the light diffusion processes in such a regime, in order to enhance the effects we described above, or to have a better control on them.

\section{Aknowledgments}

This work was partially supported by MIUR, by the INFN on the project IS-CSN4 Mathematical Methods of Nonlinear Physics and by INDAM-GNFM. 


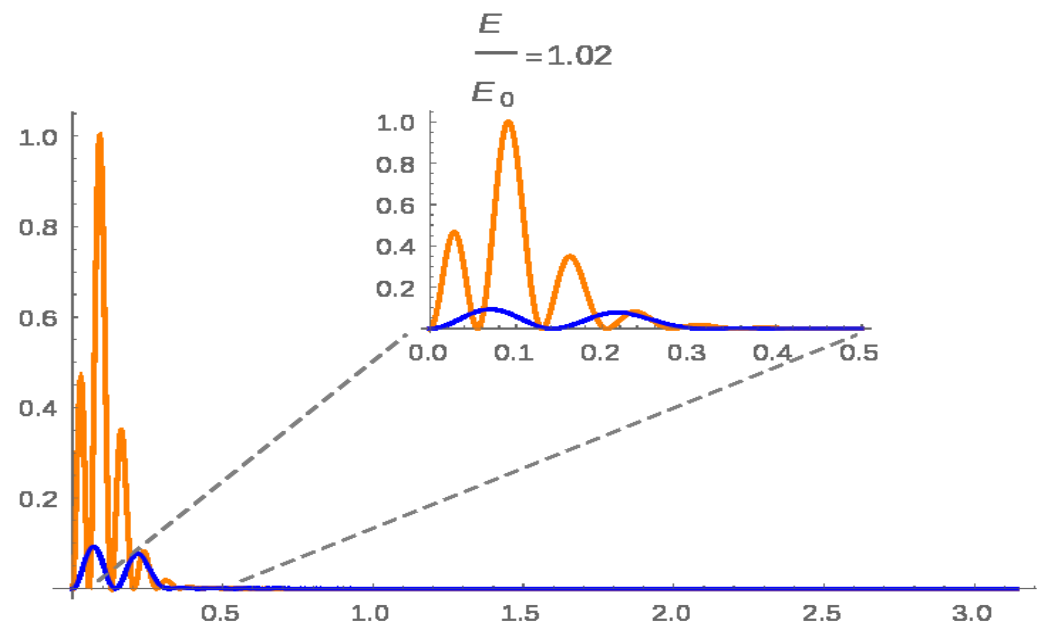

Figure 12: The numerical evaluation of the conversion cross section (4.28), in arbitrary units, for $\rho_{0}$ fixed by 2.8 and the ratio $E / E_{0}=1.02, k \rho_{0}=10$ and for the exact numerical solution (orange) and the approximated solution 2.9 (blue).

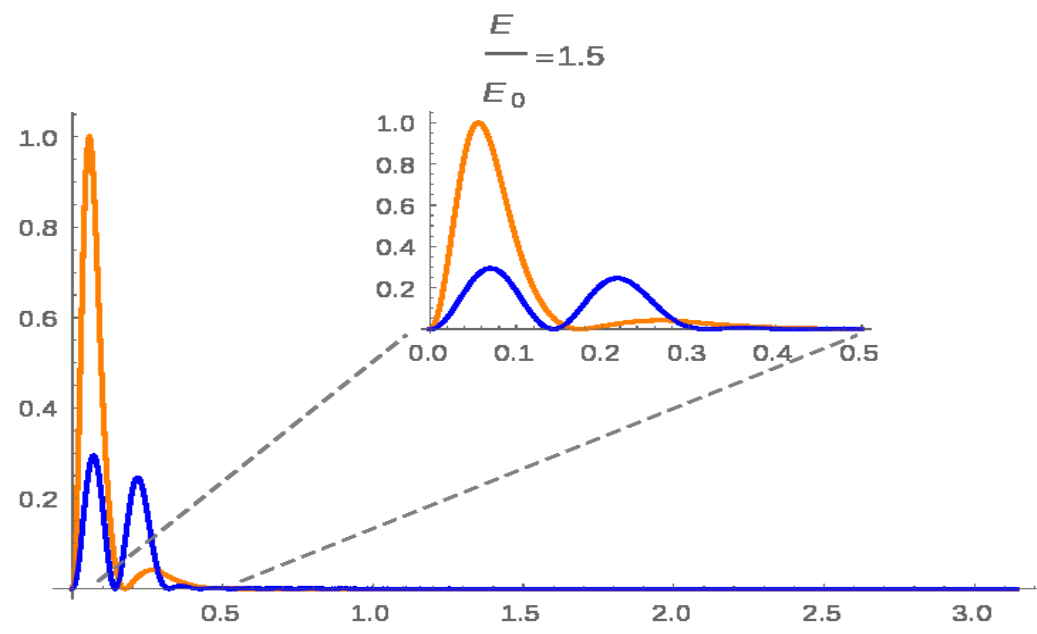

Figure 13: The numerical evaluation of the conversion cross section (4.28), in arbitrary units, for $\rho_{0}$ fixed by (2.8) and the ratio $E / E_{0}=1.5, k \rho_{0}=10$ and for the exact numerical solution (orange) and the approximated solution 2.9 (blue). 


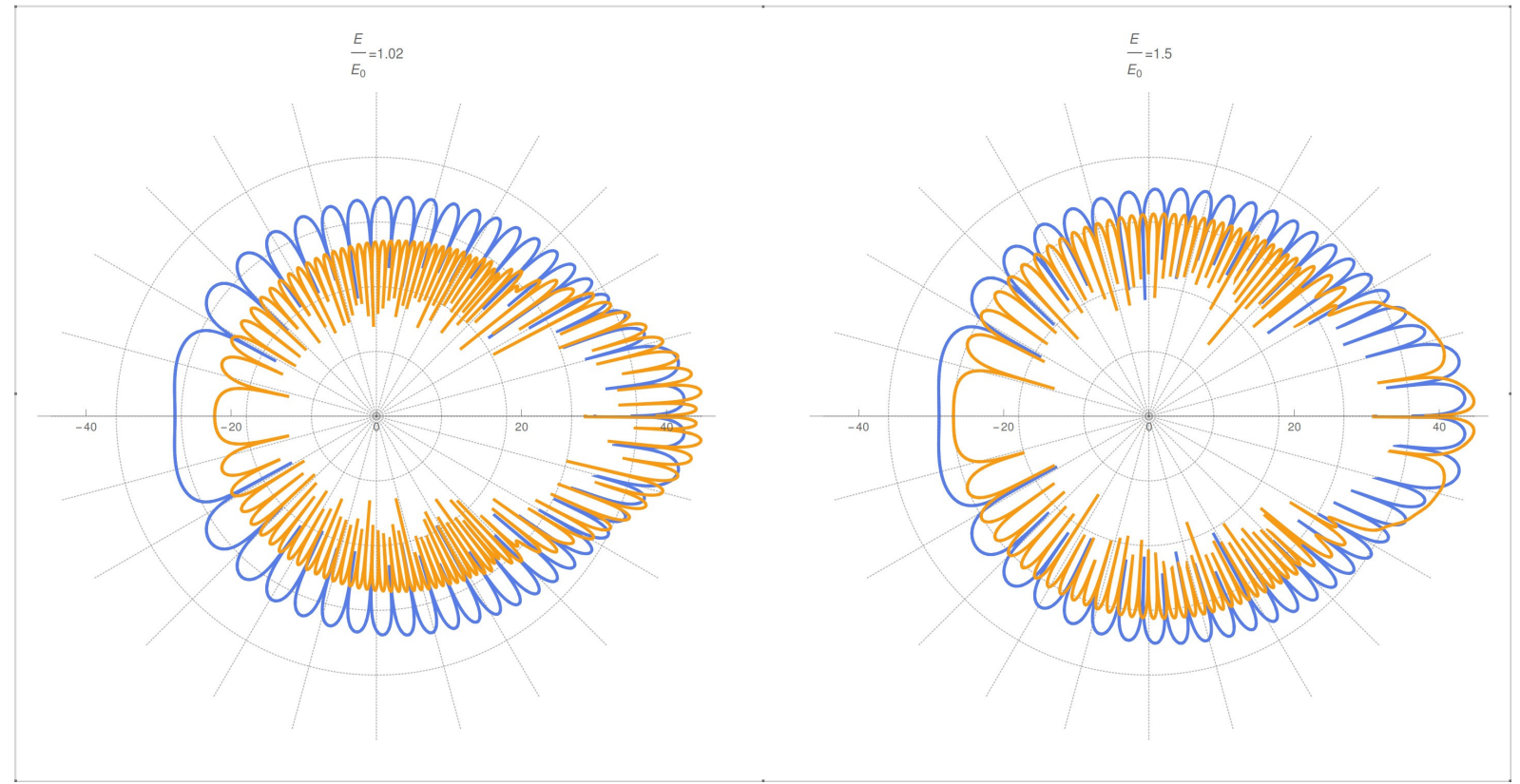

Figure 14: Comparison of the Log-polar plots of the differential cross sections for two different values of the external electric field. In both cases the orange curves refer to the exact numerical spherulite profile, while the blue ones correspond to the piecewise linear approximation of it.

\section{References}

[1] G. Luckhurst, D. Dunmur Liquid Crystals in Springer Handbook of Electronic and Photonic Materials edited by Kasap S., Capper P. (Springer Handbooks. Springer, Cham, 2017).

[2] G. V. Chigrinov, Frontiers of Optoelectronics in China 3, 103-107 (2010)

[3] H. Coles and S. Morris, Nature Photonics 4, 676-685 (2010).

[4] J. Beeckman and K. Neyts and P. J. M. Vanbrabant, Optical Engineering 50,081202 (2011)

[5] P. Oswald and J. Baudry and S. Pirkl, Phys Rep 337(1), 67-96 (2000).

[6] G. Assanto and N. F. Smyth, , IEEE Journal of Selected Topics in Quantum Electronics 22, 4400306 (2016).

[7] H.S. Kitzerow and P. P. Crooker, Liquid Crystals 11(4), 561-568 (1992).

[8] D. K. Yang and P. P. Crooker, Liquid Crystals 9(2), 245-251 (1991)

[9] Patel, Dahyabhai L. and Dupré, Donald B., Journal of Polymer Science: Polymer Physics Edition 18(7),15991607 (1980).

[10] A. O. Leonov, I. E. Dragunov, U. K. Rßler, and A. N. Bogdanov Phys. Rev. E 90, 042502 (2014).

[11] G. De Matteis and L. Martina and V. Turco, Theoretical and Mathematical Physics (to be published).

[12] J. Fukuda and S. Zumer, Nature Communications 2, 246 (2011).

[13] C. Carboni and A. K. George and A. Al-Lawati, Molecular Crystals and Liquid Crystals 410(1), 1109-1113 (2004).

[14] N. Romming, C. Hanneken, M. Menzel, J. E. Bickel, B. Wolter, K. von Bergmann, A. Kubetzka and R. Wiesendanger, Science 341, 6146 (2013).

[15] Bogdanov, A. N. and Rößler, U. K., Phys. Rev. Lett. 87, 037203 (2001)

[16] P. De Gennes and J. Prost, The physics of liquid crystals, (Clarendon Press, Oxford, 1993).

[17] I. W. Stewart, The static and dynamic continuum theory of liquid crystals: a mathematical introduction, (Taylor And Francis, 11 New Fetter Lane, London, 2004).

[18] P. Oswald, P. Pieranski, G. Gray and J. Goodby, Nematic and Cholesteric Liquid Crystals, (CRC Press, Boca Raton, 2006).

[19] R. D. Kamien and J. V. Selinger, Journal of Physics: Condensed Matter 13(3), R1-R22 (2001).

[20] T. Akahane, T. Tako Japan. J. Appl. Phys. 15, 1559 (1976). 
[21] B. Kerllenevich and A. Coche, Molecular Crystals and Liquid Crystals 68, 47-55 (1981).

[22] J. Baudry and S. Pirkl and P. Oswald, Phys. Rev. E 57, 3038 (1998).

[23] P. Oswald and A. Dequidt, Phys. Rev. E 77, 061703 (2008).

[24] S. Afghah, J.V. Selinger, Phys. Rev. E 96, 012708 (2017).

[25] A. Rapini and M. Papoular, J. Physique Colloq 30,C4 (1969).

[26] P. J. Kedney and I. W. Stewart, Letters in Mathematical Physics 31, 261-269 (1994)

[27] N. Manton and P. Sutcliffe, Topological Solitons, 1st ed, (Cambridge University Press, 2004).

[28] A. A. Belavin and A. M. Polyakov, JETP Lett. 22, 503-506 (1975).

[29] A. Barone and F. Esposito and C. J. Magee and A. C. Scott, Rivista del Nuovo Cimento 1(2), 227-267 (1971).

[30] M. J. Ablowitz and P. A. Clarkson, Solitons, nonlinear evolution equations and inverse scattering, (Cambridge University Press, 1991).

[31] B. M. McCoy. and C. A. Tracy,. and T. T. Wu, Painleve Functions of the Third Kind, J. Math. Phys 18(5) (1977).

[32] NIST Digital Library of Mathematical Functions, http://dlmf.nist.gov/.

[33] William H. Press and Saul, A., Numerical Recipes, 3rd ed., (Cambridge University Press, 2007).

[34] Randall J. LeVeque, Finite Difference Methods for Ordinary and Partial Differential Equations: Steady-State and Time-Dependent Problems, (Society for Industrial and Applied Mathematics, 2007).

[35] Jackson, J. D., Classical Electrodynamics, 3rd ed., (Wiley, 2012).

[36] M. Kleman, O.D. Lavrentovich and J. Goodby , Soft Matter Physics: An Introduction, (Springer-Verlag, New York, 2003).

[37] B. A. Lippmann, J. Schwinger, Phys. Rev. 79, 469 (1950).

[38] N. Gheorghiu, G. Y. Panasyuk, ArXiv e-prints, 1705.02683 (2017). 